\title{
LOS MEDIOS Y LA REPRESENTACIÓN DE GÉNERO: ALGUNAS PROPUESTAS PARA AVANZAR
}

\author{
PILAR LÓPEZ DÍEZ 1
}

Madrid

\section{INTRODUCCIÓN}

Quienes, con la ONU, el Consejo de Europa o la Unión Europea, consideramos que los medios de comunicación son un pilar fundamental para el logro de la igualdad entre mujeres y hombres, venimos poniendo de manifiesto desde hace décadas el desigual tratamiento que reciben los hombres y las mujeres en los medios de comunicación. Los resultados de la investigación siguen manifestando el doble rasero que mantienen quienes elaboran contenidos para los medios, no sólo la profesión periodística ${ }^{2}$. Los estudios siguen recogiendo la fijación de los medios por los roles tradicionales asociados a las mujeres, como cuando en el caso de la elección de Nancy Pelosi a la presidencia de la Cámara de Representantes de EEUU el Washington Post la describió como una «abuela de cinco nietos» dejando en el tintero una presentación más adecuada, como podría ser «una veterana con veinte años en el Congreso de EE.UU» que, suponemos, tuvo mucho más que ver con su elección. No referimos una anécdota; en el proceso postelectoral que vivió España en marzo de 2008, con un Gobierno con mayoría de mujeres y en donde la titular de Defensa estaba embarazada, la atención mediática de algunos fue puesta sobre características nada políticas de las mujeres elegidas para distintos cargos. Aunque positiva por la visibilización del problema de la categorización de las mujeres que hicieron algunos periodistas, esa atención ha puesto de manifiesto que también aquí los medios dedican demasiados recursos a destacar aspectos que nada tienen que ver con las características más importantes para la acción de gobierno o representación.

1. www.pilarlopezdiez.eu

2. Entendida no sólo como los y las periodistas, sino en un sentido amplio, que abarcaría a quienes participan, de alguna forma, en la elaboración de los contenidos tanto informativos como de programas; es decir, las y los profesionales que realizan tareas de redacción, presentación, guión, locución, documentación, corrección, producción, realización, captación de imagen y sonido y postproducción de imagen y sonido. 
Definido el problema como de falta de visibilidad para las actuaciones de las mujeres que trabajan en el espacio público, comparado con las de los hombres; de doble rasero para informar sobre los currículos de ellas y ellos, destacando de las mujeres, desproporcionadamente, su currículo familiar y, en algunos casos, de afinidad personal e ideológica con el líder ${ }^{3}$, poniendo en duda las capacidades necesarias para realizar el trabajo político; desautorizando el trabajo aún no realizado o, directamente, no mostrando ningún escrúpulo incluso en insultar a las políticas elegidas, en estos momentos debemos plantearnos algunos puntos sobre los que sería necesario reflexionar para conseguir que, poco a poco, se vayan produciendo esos cambios precisos en la elaboración de contenidos mediáticos, y su tratamiento, más acordes con el momento del siglo XXI que ya está en marcha.

\section{LA PERSPECTIVA DE GÉNERO Y LAS INICIATIVAS DE ACTUACIÓN SOBRE LOS MEDIOS}

Toda la normativa internacional, nacional o autonómica que, en forma de consejos, recomendaciones y normas (también leyes ${ }^{4}$ en nuestro país) se ha venido publicando, se ha dejado sentir en las redacciones de muchos medios a través del debate sobre la conveniencia y la necesidad, o no, de tener en consideración lo que se viene conceptualizando como implementar la "perspectiva de género» en los medios de comunicación. Perspectiva que sólo significa que los medios de comunicación también tengan en cuenta los intereses de las mujeres a la hora de elaborar sus contenidos. Aunque en otro lugar ${ }^{5}$ se ha desarrollado este concepto, podemos resumirlo:

1. Que los medios de comunicación asuman la situación de discriminación de las mujeres y las consecuencias que les han acarreado dicha posición.

2. Que cuestionen y combatan la desigualdad en que se ha situado a las mujeres respecto a los hombres, acercando a la ciudadanía las dificultades estructurales que todavía hoy encuentran las mujeres para desarrollarse en la sociedad.

3. Que observen y comprendan cómo opera la discriminación negativa hacia las mujeres (invisibilizándolas y minimizando sus logros y aportaciones); y la discriminación positiva hacia los hombres (destacando en sus prácticas profesionales las actuaciones masculinas e invisibilizando o justificando comportamientos indeseados, incluso punibles, de los hombres).

4. Que colaboren, de forma activa y comprometida, en la erradicación de dicha desigualdad (en las redacciones, eligiendo idóneamente al

3. El caso de Sáenz de Santamaría respecto a Rajoy.

4. Ley de Medidas integrales contra la violencia de género, Ley de Igualdad efectiva de mujeres y hombres.

5. LÓPEZ DíEZ, Pilar: «¿Cómo tratan la violencia de género los medios de comunicación?» en J. Plaza y C. Delgado (eds.): Género y Comunicación, Madrid, Editorial Fundamentos, 2007, pp. 73-99. 
personal responsable, sin discriminación de sexo, y dedicando medios y recursos para crear las condiciones precisas para que los medios de comunicación contribuyan a que las mujeres desarrollen su potencial humano y su autonomía; es decir, para que tomen el control de su vida en todos los ámbitos, sin la tutela masculina).

En las redacciones la perspectiva de género entró principalmente de la mano de mujeres involucradas y sensibilizadas de las redacciones de informativos, en forma de discusiones, muchas veces agrias, sobre el lenguaje sexista y la conveniencia de superar el genérico masculino para visibilizar a las mujeres; de propuestas para cubrir temas que consideraban importantes para que toda la sociedad las conociera, como las agresiones sexuales o el acoso laboral, y que los editores y redactores-jefes no valoraban como noticiable; de debates a raíz de las críticas de algunas lectoras, oyentes o telespectadoras sobre lo que consideran comentarios inadecuados o posiciones sesgadas sobre actuaciones o iniciativas de candidatas a algún puesto de responsabilidad.

Ha habido también propuestas y debates promovidos por periodistas, mayoritariamente mujeres, dentro de organizaciones profesionales como la FAPE, para mejorar el tratamiento de las mujeres en los medios, especialmente en los temas de violencia de género, a nivel organizativo ${ }^{6}$. También sobre este asunto, en prácticamente todas las autonomías, incluso en algunas provincias, se han promovido propuestas para que los medios mejorasen el tratamiento de las noticias de violencia de género ; la experiencia ha ido más allá y organizaciones sociales y de mujeres ${ }^{8}$ también han publicado sus propias recomendaciones.

La actividad y organización que están desplegando desde hace años algunas periodistas comprometidas en la mejora del tratamiento de la igualdad en las noticias dio lugar al I Encuentro Nacional de Mujeres Periodistas por la Igualdad que tuvo lugar en Granada ${ }^{9}$ y cuyos objetivos fueron analizar la situación de la profesión desde la perspectiva de género y el lugar que ocupan las mujeres en los distintos medios de comunicación, por un lado, y estudiar la repercusión que tiene el trabajo de las periodistas en la vida cotidiana de las españolas y en qué medida se contribuye o no a la resolución de problemas, por otro.

Con la finalidad de hacer llegar a una audiencia amplia el problema de la representación de las mujeres y los hombres en los medios de comunicación, también se han llevado a cabo numerosos actos, organizados por instituciones

6. "Manual para periodistas sobre la violencia doméstica: Noticias con lazo blanco», promovido por la Unión de Periodistas de Valencia, 2002. "Tratamiento de la violencia contra las mujeres en los medios de comunicación. Recomendaciones para las buenas prácticas en la información sobre violencia de género", 2005, coordinado por mujeres periodistas de las asociaciones de la prensa de Ciudad Real, Granada, Aragón y La Rioja.

7. Tienen sus propias recomendaciones Andalucía (RTVA), Cataluña (CAC), Islas Baleares, Aragón, Comunidad Valenciana y otras autonomías, además de, a nivel estatal, RTVE. En todos los casos se trata de normas que no son de obligado cumplimiento para quienes elaboran los contenidos.

8. Como la Federación de Mujeres Progresistas.

9. Celebrado el 8 y 9 de febrero de 2008, organizado por la Agrupación de Mujeres Periodistas de Granada y que reunió a más de doscientas personas. 
estatales, autonómicas, locales y provinciales, como Institutos o Direcciones Generales de la Mujer, diputaciones o ayuntamientos que vienen desarrollando un amplio calendario de jornadas, cursos y seminarios, y que, a través de personas expertas en diferentes materias, han acercado este asunto no sólo a profesionales del periodismo sino también, y con gran acierto, al público en general, mayoritariamente femenino, que es quien asiste a este tipo de actos.

Podemos concluir que gracias a estas iniciativas ha llegado a determinados sectores (los más preocupados y sensibles con la situación de desigualdad de las mujeres, además de quienes, en muchos casos por exigencias de su trabajo en organismos de igualdad se ven obligadas a seguir cursos de capacitación sobre distintos temas) el problema que significa la escasa atención que dispensan los medios de comunicación a la desigualdad de género.

\section{PROTOCOLO DE ACTUACIÓN PERIODÍSTICA Y PUBLICITARIA SOBRE IGUALDAD DE OPORTUNIDADES ENTRE MUJERES $Y$ HOMBRES $Y$ TRATAMIENTO INFORMATIVO DE LA VIOLENCIA DE GÉNERO}

Uno de los primeros trabajos sobre cómo deberían cubrirse los hechos violentos protagonizados por los hombres, que daban lugar a las noticias sobre violencia de género, el Manual de Urgencia para el tratamiento de las noticias de violencia de género (2002) de RTVE, abría su decálogo con el siguiente texto: «1. [Debemos] evitar los modelos de mujer que lesionen su dignidad. La industria de la cultura difunde un modelo de mujer que atenta contra su dignidad (figura vicaria, objeto, subyugada). Haz lo posible por evitar esos estereotipos». Y añadía: "Hay que ampliar la representación de las mujeres en los medios para evitar su victimización. También hay mujeres ingenieras, transportistas, pintoras, mineras, médicas...».

Se recogía así, el punto fundamental sobre el que debería pivotar el cambio en el tratamiento y la elaboración de contenidos que afectasen a las mujeres, a los hombres y a sus relaciones por parte de los medios de comunicación. No era posible, se argumentaba en el trabajo ${ }^{10}$, seguir construyendo las mismas representaciones sobre las mujeres, basadas en estereotipos y roles secundarios por un lado, e intentar luchar contra la violencia de género efectivamente desde los medios de comunicación, por otro. La violencia estructural que sufren las mujeres se alienta y promueve desde la sociedad patriarcal que todavía no respeta a las mujeres y lo femenino como lo hace con los hombres y lo masculino; pero también desde los medios de comunicación que tanto entonces, como ahora, apenas reflejan las aportaciones de las mujeres a la sociedad.

Es en 2007 cuando el Gobierno de Cantabria, a través de la Dirección General de la Mujer, tiene la iniciativa de promover el «Protocolo de actuación periodística y publicitaria sobre igualdad de oportunidades entre mujeres y hombres

10. López DíEz, Pilar: "La violencia contra las mujeres en los medios de comunicación», en I Foro Nacional: Mujer, violencia y medios de comunicación, Madrid, Instituto Oficial de Radio y Televisión (RTVE) e Instituto de la Mujer (Ministerio de Trabajo y Asuntos Sociales), 2002, pp. 21-40. 
y tratamiento informativo de la violencia de género $»^{11}$ en el que se articulan 38 recomendaciones para mejorar el tratamiento no sólo de la violencia de género, sino de todas aquellas informaciones en donde estén involucradas mujeres y hombres. Con esta propuesta a los medios de comunicación de aquella comunidad autónoma, se trataba de avanzar un paso más en el diagnóstico habitual de las representaciones mediáticas en donde se venía diciendo que tenían que superar las imágenes estereotipadas y los roles de género tradicionales. Así, en el punto primero, en donde se apunta a la necesidad de visibilizar a las mujeres y sus logros y aportaciones a la sociedad actual, ampliando su campo de representación y haciéndolo de forma justa, se especifican siete formas de lograrlo; entre otras, a través de solicitar y contar con las voces de las mujeres, de la misma forma que se hace con las de los hombres. Se sugiere que se eviten las descripciones físicas cuando son mujeres las protagonistas de la información, también las referencias sexistas ${ }^{12}$, los estereotipos degradantes y las frases condescendientes. Se recomienda superar los estereotipos sexuales considerando noticiables también aquellos acontecimientos protagonizados por mujeres alejadas del prototipo imperante.

El Protocolo desaconseja sexualizar a las niñas, que lo son hasta los catorce años, a través de una representación inadecuada en esta edad, y que las coloca en situación de dependencia y sumisión respecto a sus pares y a los hombres; se menciona también la necesidad de superar el estereotipo del varón falto de sentimientos ilustrando asuntos emotivos y de interés humano con imágenes de hombres que sufren. Se recomienda no reforzar la consabida deslealtad, competencia y enemistad entre mujeres que refuerza el estereotipo interesado de que "la mayor enemiga de una mujer es otra mujer» y se recomiendan prácticas de comportamiento ético que evitan la utilización del doble rasero, destacando y censurando acciones reprobables sólo cuando están protagonizadas por mujeres: «Una mujer abandona a su hijo de cuatro años en un locutorio», dice una titular $^{13}$, obviando la existencia de la responsabilidad paterna.

El Instituto Oficial de Radio y Televisión (RTVE) organizó un Congreso Nacional que tituló "Televisión y políticas de igualdad»" en donde presentó una «Propuesta de actuación para la televisión en materia de igualdad entre hombres y mujeres» que destaca dieciséis recomendaciones en los siguientes apartados: Prácticas generales en informativos, programas y ficción. La violencia de género. Prácticas internas en la organización y prácticas supraorganizacionales, en donde se mencionaba la necesidad de facilitar foros de diálogo con los operado-

11. Vicepresidencia, Dirección General de la Mujer, Gobierno de Cantabria. 2007. Puede consultarse en Publicaciones: www.pilarlopezdiez.eu

12. En el contexto de una entrevista en donde se quiere saber qué ideas tiene la nueva ministra respecto al trabajo a desarrollar en el ministerio ¿es pertinente terminar la entrevista preguntándole si está soltera?

13. El País, 12 de diciembre de 1999.

14. Madrid, 16 y 17 de octubre de 2007, junto con el Instituto de la Mujer. Texto en Publicaciones: www.pilarlopezdiez.eu 
res de televisión, productoras de contenidos, guionistas, personal investigador, asociaciones de mujeres y otros agentes como la Administración.

Pero las iniciativas destacadas hasta este punto son recomendaciones, guías, consejos que se ofrecen a quienes tienen la responsabilidad social de elaborar contenidos en los medios, sin que ninguna de ellas sea de obligado cumplimiento. No es extraño, pues, que muchas y muchos de quienes trabajan en los medios que han suscrito cualquiera de estas propuestas ni siquiera conozcan su contenido. ¿Queremos decir, entonces, que no sirve de nada el camino recorrido hasta el momento? No. Atendiendo al efecto sobre los propios medios de comunicación, a sus intereses económicos y corporativos no se les hace ningún favor al publicitar cualquier iniciativa que critica y pone en tela de juicio prácticas discriminatorias contra las mujeres. Además, el efecto de estas propuestas alcanza a un segmento del personal que trabaja en los medios que sí tiene y se exige un grado de compromiso con la igualdad. Estas periodistas y algunos trabajadores más encuentran legitimadas sus posiciones que, seguramente, fueron rechazadas y puestas en tela de juicio por sus jefes antes de que se visibilizara el tratamiento de la igualdad de género en los medios. Por otra parte, no sería posible entrar en la siguiente etapa, la autorregulación, si no se hubiese recorrido la que acabamos de señalar.

\section{EL DECÁLOGO DEL DIARIO PÚBLICO Y EL ESTATUTO DE INFORMACIÓN DE LA CORPORACIÓN RTVE}

En 2008 se están dando las condiciones para superar la práctica que hemos descrito hasta el momento; habría sido imposible haber llegado al momento actual si no se hubiese transitado por las etapas anteriores de voluntariedad, y asistido a la presentación de una auténtica inflación de normas y recomendaciones para los medios a nivel estatal, autonómico o local. Como consecuencia de las políticas de igualdad promovidas también a todos los niveles en nuestro país, y de la singular situación en que nos encontramos respecto a nuestro entorno cultural, con leyes orgánicas que se han ido aprobando y que se están implementando, se ha desarrollado una iniciativa destacable en el diario Público. Desde comienzos de enero de 2008, cuando se conoció su compromiso, este periódico ha adoptado un «Decálogo para informar sobre violencia de género» ${ }^{15}$ en el que manifiestan: "A partir de ahora, desde la redacción de Público nos comprometemos a que nuestro decálogo sea de obligado cumplimiento y pedimos a nuestros lectores que así nos lo exijan». Este es un nivel de compromiso que afecta a la redacción y que obliga a sus profesionales a seguirlo. El público de Público está obligado a hacer un seguimiento de sus contenidos respecto al ámbito sobre el que se comprometen, de manera que sus lectoras y lectores han adquirido también el compromiso con su periódico para velar por una información respetuosa con la igualdad. El punto más importante que define

15. http://www.publico.es/039014/publico/compromete/decalogo/ionformar/violencia/genero 
este Decálogo es el quinto ${ }^{16}$ : "Nunca buscaremos justificaciones o "motivos» (alcohol, drogas, discusiones...). La causa de la violencia de género es el control y el dominio que determinados hombres ejercen contra sus compañeras».

Más recientemente ${ }^{17}$ fue aprobado por la mayoría de la plantilla que interviene en la elaboración de información el Estatuto de la Información de la Corporación RTVE; más del setenta por cierto de quienes votaron lo hicieron a favor y de esta forma estuvieron de acuerdo con el contenido del Código deontológico, en donde, entre otras prácticas éticas contempladas en muchos estatutos y códigos, como el tratamiento de las fuentes informativas, derecho de rectificación o el tratamiento que afecte a menores, se recoge:

"Valorarán con el mismo criterio las acciones protagonizadas por mujeres y hombres a la hora de considerarlas noticiables, y emplearán similares recursos técnicos y estéticos en su elaboración. Reflejarán adecuadamente la presencia de las mujeres en los diversos ámbitos de la vida social y evitarán el uso de referencias sexistas y estereotipos degradantes» ${ }^{18}$.

Este importante punto, probablemente, será muy beneficioso para implementar el principio de la «ampliación del campo de representación de las mujeres» del que se hablaba anteriormente y, al mismo tiempo, debería influir en la superación de la representación estereotipada de las mujeres y los roles de género a ellas asignados. Como consecuencia de este punto, esperemos que, a partir de ahora, se informará y celebrarán de la misma forma los éxitos de las mujeres y de los hombres, corrigiendo la práctica habitual de destacar casi sólo los masculinos ${ }^{19}$. Sin embargo, habría sido muy conveniente que, además de comprometerse a que "se emplearán similares recursos técnicos y estéticos en su elaboración», se hubiese hecho mención, también, a que se dedicarían iguales recursos humanos ${ }^{20}$.

En el art. 37. de la Ley de Igualdad, dedicado a la Corporación RTVE, se recoge que ésta, en el ejercicio de su función de servicio público, perseguirá en su programación varios objetivos, uno de los cuales es «Reflejar adecuadamente la presencia de las mujeres en los diversos ámbitos de la vida social». Este objetivo se ha plasmado en el punto destacado anteriormente, por el que nos felicitamos. Sin embargo, notamos la ausencia del segundo objetivo recogido en dicho artículo: "Utilizar el lenguaje en forma no sexista». Tampoco se ha intro-

16. Entre los comentarios que generó la publicación del Decálogo en Público, la mayoría de los cuales eran de lectores, este fue uno de los puntos que más rechazo despertó entre quienes criticaban la iniciativa -aproximadamente se decantaron a favor de la propuesta la mitad de quienes enviaron comentarios.

17. 18 de abril de 2008 .

18. http://periodismoglobal.files.wordpress.com/2008/03/estatuto-de-informativosrgltofinal.doc, p. 5.

19. Las deportistas entrevistadas en las 880 noticias analizadas fueron 3 ; los deportistas a quienes se les dio voz fueron 153. López DíEZ, Pilar: Representación de género en los informativos de radio y televisión, Madrid, IORTVE e Instituto de la Mujer, 2005.

20. Sería de justicia que se enviase el mismo número de periodistas y de cámaras para cubrir los europeos de Baloncesto masculino y femenino. 
ducido el punto 2 del mismo artículo de la misma Ley: «[La Corporación RTVE] fomentará la relación con asociaciones y grupos de mujeres para identificar sus necesidades e intereses en el ámbito de la comunicación", que interpretamos como un paso necesario a seguir en la implementación del derecho de acceso para los grupos sociales significativos que recoge de manera exhaustiva la vigente Ley de la radio y la televisión de titularidad estatal ${ }^{21}$ a la que está obligada la Corporación.

Sobre el tratamiento de las noticias de violencia de género, el Código deontológico de RTVE recoge: «Dedicarán especial atención a las informaciones relativas a la violencia de género, evitando la transmisión de mensajes que puedan contribuir a crear en la sociedad sensación de impunidad ante estos delitos». El "Manual de Urgencia» para el tratamiento de las noticias de violencia de género y el reciente documento "Televisión y políticas de igualdad» ${ }^{22}$ han desarrollado más exhaustivamente este importante tema en la medida en que la Corporación RTVE se ha venido posicionando claramente en la denuncia de la violencia de género.

De la misma forma que en el caso del Decálogo de Público, el Código deontológico de la Corporación RTVE es una norma de obligado cumplimiento para todas aquellas personas que trabajen en Informativos.

\section{ALGUNAS PROPUESTAS}

\subsection{La formación y competencia de quienes elaboran contenidos mediáticos y publicitarios}

Entre las 80 propuestas de actuación que contempla el primer informe sobre la evolución de la violencia de género y de la efectividad de las medidas acordadas para la protección de las víctimas recogidas por el Observatorio Estatal de Violencia sobre la Mujer ${ }^{23}$ hay siete que se refieren a los medios de comunicación. Entre otras, se incluye que haya en las redacciones y en las jefaturas de redacción periodistas con especialización en temas de género, capaces de aplicar enfoques no androcéntricos a las noticias y reportajes.

De la misma forma que se aconseja para quienes trabajan en cualquier otro sector como la sanidad, la abogacía, la judicatura, la asistencia social o cualquier otro la formación en género también es imprescindible para los medios de comunicación para informar en general y para implementar adecuadamente las políticas de igualdad que se promuevan desde las diferentes administraciones en particular. Como señala el documento de RTVE ${ }^{24}$ :

21. Ley $17 / 2006$ de 5 de junio.

22. Televisión y políticas de igualdad, Madrid, Instituto Oficial de Radio y Televisión e Instituto de la Mujer (Ministerio de Trabajo y Asuntos Sociales), 2007.

23. Primer Informe Anual del Observatorio Estatal de Violencia sobre la Mujer. 13 de julio de 2007, en: http://www.observatorioviolencia.org/upload_images/File/DOC1184747918_Informe\%20 Anual\%2028\%20junio\%202007.pdf, pp. 204-205.

24. Televisión y políticas de igualdad, op.cit., p. 9. 
«La formación es fundamental para difundir una imagen igualitaria de la mujer, que deje atrás prácticas sexistas y discriminatorias, conscientes o inconscientes, fruto de siglos de educación machista, por eso considera que es preciso: "Sensibilizar, mediante la formación, a los y las profesionales de la comunicación y a los cargos directivos de las televisiones y productoras sobre el principio de igualdad entre mujeres y hombres».

Desde el año 2001 se han llevado a cabo cursos de formación en RTVE ${ }^{25}$, para periodistas de distintos medios en Asturias ${ }^{26}$, en Ciudad Real, Huesca y otras localidades; y en Cartagena de Indias, para periodistas de Iberoamérica, se ofreció un curso intensivo de una semana de duración ${ }^{27}$.

La experiencia obtenida en este campo se puede resumir:

5.1.1. Aquellas personas con experiencia profesional en los medios, sin conocimientos sobre comunicación y género y que asisten voluntariamente a los cursos aunque tienen muchas dificultades para asegurar la asistencia, en principio, ofrecen cierta resistencia a cambiar las rutinas habituales, pero terminan valorando las herramientas que se les ofrecen $\mathrm{y}$, con posterioridad, las introducen en sus rutinas profesionales: proponen y elaboran nuevos temas que antes no se les habían ocurrido; enriquecen temas ya desarrollados y cuentan con otro tipo de fuentes de las que prescindían con anterioridad.

5.1.2. La formación en género para quienes todavía están en proceso de formación en las facultades de periodismo supone un descubrimiento y aceptan, con menor resistencia, las normas deontológicas y las prácticas en que se basa la perspectiva de género.

En cualquier caso, habría que avanzar en este punto de la formación; sería necesario que, expresamente, desde los cargos directivos de los medios se comprometieran con la causa de la igualdad de género y apoyasen, en la práctica, la formación del personal, de manera que fomenten la asistencia facilitando el horario adecuado; acercando los cursos de formación al personal interesado y considerando como formación profesional el seguimiento de dichos cursos, además de valorar como méritos (para ascender en los puestos de responsabilidad, elección de cargos directivos, etc.) la asistencia a los cursos de formación en género y comunicación.

Quienes son propietarios, dirigen u ostentan puestos de decisión y responsabilidad en los medios y obvian las propuestas de formación en género para sus profesionales desconocen que la perspectiva de género desarrolla sus capacidades, talento y creatividad, consiguiendo contenidos y desarrollos menos clónicos y más originales, que interesan a un espectro más amplio que su público objetivo y diferencian sus productos de los de quienes siguen los estándares tradicionales. Este enfoque debería interesar especialmente en el momento en

25. Organizados por el Instituto Oficial de Radio y Televisión y el Instituto de la Mujer.

26. Instituto Asturiano de la Mujer (2003).

27. Instituto de la Mujer, AECI e Instituto de la Mujer (2003). 
que nos encontramos con la segmentación de audiencias que puede provocar las tecnologías de la información y la comunicación.

La resolución 1557 de la Asamblea Parlamentaria del Consejo de Europa ${ }^{28}$, respeto a la Publicidad sexista, sigue insistiendo en la importancia de la formación para quienes trabajan en agencias de publicidad, y denuncia que demasiado a menudo éstas muestran a las mujeres en situaciones que son humillantes y degradantes o, incluso, -añade- violentas y ofensivas para la dignidad humana; esta resolución aboga por que el respeto a la dignidad humana debería ser uno de los objetivos constantes de los publicitarios y les recomienda seguir cursos de capacitación sobre igualdad para evitar la discriminación.

\subsection{Estudios reglados sobre comunicación y género en las Facultades de Ciencias de la Información y la Comunicación}

Quienes creen que la práctica de un periodismo no sexista y respetuoso con las mujeres va a ser su profesión futura tienen el derecho a exigir en los planes de estudio que van a cursar que se impartan aquellos conocimientos sobre género que han estado ausentes en los currícula universitarios hasta el día de hoy. De la misma forma que se ha recogido como buena práctica en otros países europeos $^{29}$, sería conveniente contemplar los estudios de género para disciplinas involucradas directamente en la igualdad y en la violencia de género como en Comunicación, Derecho, Medicina, Trabajo Social, etc.

También en el Primer Informe Anual sobre la evolución de la violencia masculina contra las mujeres, del Observatorio Estatal de Violencia sobre la Mujer, se recogen ocho propuestas en el ámbito universitario; una de ellas explicita que se «incluyan materias especializadas en género en todas las titulaciones de grado» que deberán diseñarse en las universidades a partir de $2007 »^{30}$.

Los planes de estudio que incluyan la perspectiva de género y el compromiso del profesorado de comunicación, tal como señala Cristina Segura ${ }^{31}$ para cualquier materia, podrían propiciar el cambio de mentalidades entre quienes tendrán en sus manos la información futura:

"La incidencia en la docencia es imprescindible pues a través de las enseñanzas impartidas a todos los niveles es donde puede modificarse la mentalidad dominante

28. «La imagen de las mujeres en la publicidad», de 26 de junio de 2007.

29. «En Suecia, dentro de los temas sobre igualdad de género, que forman parte de los requisitos para el examen obligatorio (a nivel universitario) de Derecho, Medicina, Enfermería, Psicología, Psicoterapia, Trabajo Social, Cuidado Social y Teología, se estudia la violencia contra las mujeres en el currículo universitario obligatorio" (ESPAÑA 2002. Presidencia de la Unión Europea. Ue2002.es. "Guía de buenas prácticas para paliar los efectos de la violencia contra las mujeres y conseguir su erradicación", p. 79).

30. Informe anual del Observatorio Estatal de Violencia sobre la Mujer, en: http://www.observatorioviolencia.org/upload_images/File/DOC1184747918_Informe\%20Anual\%2028\%20 junio\%202007.pdf, p. 203.

31. Segura Graíño, Cristina: «III. La docencia y los estudios de las mujeres, feministas y de género en la Universidad", en T. Ortiz y otras: Universidad y feminismo en España (II), Granada, Instituto de Estudios de la Mujer de la Universidad de Granada, 1999, pp. 135-185. 
sobre las mujeres y cambiarse los comportamientos sociales. La enseñanza en la igualdad es la clave para lograr erradicar los planteamientos patriarcales, todavía presentes con más o menos fuerza en las sociedades actuales. Por eso, una docencia en la que estén presentes las aportaciones de los estudios de las mujeres es una pieza imprescindible para lograr el cambio social».

Es preciso destacar la labor que están realizando algunas profesoras desde los estudios de postgrado ${ }^{32}$ en las universidades españolas en los que diseñan módulos que contemplan la igualdad desde los medios de comunicación. La Universidad Pontificia de Salamanca puso hace dos cursos académicos en marcha un postgrado en Comunicación y género.

\subsection{El papel de la audiencia y, especialmente, el de las organizaciones de mujeres y feministas: la transformación de la audiencia desinformada en un público crítico y exigente}

Además de la formación en la perspectiva de género de quienes trabajan en los medios y de la necesidad de tenerla en cuenta desde los planes de estudio universitarios, es preciso fortalecer el papel de la audiencia como receptora responsable del proceso de producción de los medios, ya que, tal como señala el Código Europeo de Deontología del Periodismo, es la propietaria, en última instancia, de la información.

En abril de 2007 se presentó a los medios la Comisión Asesora de la Imagen de las Mujeres en la Publicidad y en los Medios de Comunicación, presidida por el Instituto de la Mujer y que está formada por representantes de todos los operadores de radio y televisión tanto públicos como privados, por distintas asociaciones de anunciantes, agencias de publicidad, periódicos y revistas, y AUTOCONTROL. Además de organismos estatales como el Instituto Nacional de Consumo o la Secretaría de Estado de Comunicación, también pertenece a dicha Comisión la Asociación de Dones Periodistes de Catalunya. Entre las funciones de dicha Comisión está la de asesorar al Observatorio de la Imagen de las Mujeres del Instituto de la Mujer, y proponer y colaborar en la realización de acciones a favor de la imagen de las mujeres en la publicidad y en los medios de comunicación. Otra de sus funciones es promover la autorregulación y la colaboración entre las entidades firmantes dentro de los sectores de la publicidad y los medios de comunicación para el correcto tratamiento de la imagen de las mujeres.

En este contexto creemos que es preciso tener también en cuenta a la audiencia formada e informada, y especialmente, a las mujeres como activistas que quieren ser partícipes activas, ayudando a los medios de comunicación y a la industria publicitaria en el importante papel que tienen que desarrollar respecto a la igualdad de género. Una audiencia formada, informada y organizada debe hacerse oír y debe tener un papel relevante en el logro de los objetivos

32. En las universidades de A Coruña, Complutense de Madrid, Castilla-La Mancha (CEU de Talavera de la Reina) y la Facultad de Psicología de la Pontificia de Salamanca, entre otras. 
antes señalados. La existencia de asociaciones de mujeres usuarias de medios de comunicación y de publicidad es necesaria para equilibrar los intereses económicos y corporativos de quienes producen los contenidos y los distribuyen, y deberían participar, en igualdad de condiciones, en los organismos que se creen o se puedan crear y que traten sobre la imagen de las mujeres. Por eso es preciso atender a la alfabetización audiovisual de la audiencia.

En Madrid y también en Castilla-La Mancha, con distinto nivel de desarrollo, se está implementando un proyecto titulado «Formación de formadoras en comunicación desde la perspectiva de género para la sensibilización y dinamización de asociaciones, entidades y organizaciones de mujeres» cuyo objetivo es la formación de ese público informado y crítico. El proyecto parte de la formación desde la perspectiva de género de algunas periodistas o licenciadas en periodismo de cada localidad; después de un curso de inmersión de 30 horas, se reúnen con las asociaciones de mujeres en sesiones quincenales en donde analizan el contenido de noticias de actualidad, tanto de prensa como radio o televisión, y otros relatos procedentes de la ficción o de la publicidad. En cada caso, si se considera necesario, se elabora un texto, en donde se recogen los argumentos desde la perspectiva de género, dirigido al medio que ha publicado o emitido el mensaje original. Por último, si así se decide, se recogen firmas entre las socias y personas relacionadas para enviarlas, junto con el comentario y su posición, al medio correspondiente.

Abogamos por que la alfabetización audiovisual llegue a las asociaciones de mujeres, feministas y, en general, organizaciones sociales, para que puedan intervenir para evitar la publicidad sexista y violenta tal como señala la resolución 1557 de la Asamblea Parlamentaria del Consejo de Europa, que recomienda actuar en tres frentes: regulación, autorregulación y formación para ayudar a la gente a reaccionar críticamente ante la publicidad.

\subsection{El pluralismo y el derecho de acceso}

En España se ha entendido el derecho de acceso a los medios de titularidad pública, en la práctica, como el ofrecimiento de un tiempo determinado de emisión, los conocidos espacios electorales, a los partidos y grupos políticos para que, en las convocatorias electorales, difundiesen sus programas. La Ley $17 / 2006$ de la radio y la televisión de titularidad estatal en su artículo 28 contempla este importante derecho cuyo ejercicio aún no se ha regulado. Dicho artículo recoge que la Corporación RTVE asegurará en su programación la expresión de la pluralidad social, ideológica, política y cultural de la sociedad española, y que se aplicará de manera global, mediante la participación de los grupos sociales y políticos significativos, como fuentes y portadores de información y opinión en el conjunto de la programación de RTVE; y de manera directa, mediante espacios específicos en la radio y la televisión con formatos diversos, tiempos y horarios, fijados en el Consejo de Administración; además se garantizan la disponibilidad de medios técnicos y humanos necesarios para la realización de los espacios para el ejercicio del derecho de acceso. 
Como señala una experta ${ }^{33}$ es necesario generar estrategias para desarrollar una conciencia comunicacional en la ciudadanía, que significa conocer la relevancia de la dimensión de la comunicación para las organizaciones sociales de hoy, y reconocer que es un elemento constitutivo de poder y decisión, organización y producción de significado en los espacios donde se define lo público y lo privado. Las organizaciones de mujeres y aquellas que específicamente puedan plantear su trabajo hacia los medios, y, en general, las organizaciones sociales sensibles con los problemas de representación, deben aprovechar los espacios que contempla la ley para hacer oír sus propuestas.

\subsection{La visibilización del estatus de quienes se comprometen con la igualdad}

Como señala el Consejo de Europa es importante que las instituciones de igualdad que se creen dependan de los más altos niveles políticos ${ }^{34}$; de la misma forma podríamos decir que la implicación personal, real, pero también simbólica, a través de su representación en los medios, de quienes ocupan los más altos puestos de responsabilidad en el país ${ }^{35}$ podría ayudar a conseguir el objetivo de la igualdad. En abril de 2008 el lendakari Ibarretxe firmó, junto con otros 26 vascos, la "Carta de los hombres vascos por la igualdad y contra la violencia hacia las mujeres»; todos los medios de comunicación autonómicos se hicieron eco del acto; pero no sólo se recogió como noticia el acontecimiento, también en dos periódicos de los de mayor circulación en el País Vasco se publicaron sendos editoriales comentando y apoyando la medida. Pocos días después, en hora de máxima audiencia de La Primera de TVE, el presidente del Gobierno hablaba sobre las ministras recientemente elegidas y decía:

«El mayor retraso histórico que ha tenido España era la marginación, desigualdad y dominación de las mujeres. Los países donde hay más bienestar son aquellos donde existen una igualdad real en todos los ámbitos».

Las mismas palabras se recogieron en varios periódicos. El estatus de quienes manifiestan ideas a favor de la igualdad tiene influencia en la percepción social.

Los hechos, a través de la implementación de pertinentes políticas de igualdad, son imprescindibles; pero la responsabilidad de los medios como creadores

33. UCA SILVA, Teresa: «Beyond Contents: Proposals From a Gender Perspective For Intervention In the Media». Expert Group Meeting on «Participation and access of women to the media, and the impact of media on, and its use as an instrument for the advancement and empowerment of women», ONU, Beirut, Líbano, 12 al 15 de noviembre de 2002.

34. TAVARES DA SILVA, Maria Regina: Stocktaking study of the effective funtioning of nacional mechanisms for gender equality in Council of Europe member states, Bruselas, Dirección General de Derechos Humanos, 2004.

35. Las habituales declaraciones sobre las políticas de igualdad del Presidente del Gobierno, Rodríguez Zapatero; o las intervenciones del portavoz del Gobierno en el Congreso, José Antonio Alonso, evitando el genérico masculino y visibilizando a las españolas de la misma forma que a los españoles, son una muestra. 
de opinión, cubriéndolos adecuadamente y colocándose claramente a favor de las iniciativas por la igualdad, especialmente a través de sus editoriales, artículos de opinión, reportajes interpretativos, etc. lo son aún más. La agenda política determina en buena medida la agenda mediática, y ésta, a su vez, influye en la agenda social, acercando a la ciudadanía la información y los posicionamientos de quienes, desde las más altas responsabilidades de gobierno, han sido elegidas o elegidos para representarla.

\section{CONCLUSIONES}

Primera: Las políticas públicas sobre igualdad que se vienen desarrollando en nuestro país han influido para que dentro de los medios de comunicación se haya desarrollando una corriente que, con mayor o menor interés, actividad y compromiso, pone en cuestión ciertos aspectos de la representación de las mujeres que habían sido ignorados hasta fechas relativamente recientes.

Segunda: Las numerosas y variadas propuestas de recomendaciones, consejos y buenas prácticas sobre el tratamiento informativo de la violencia de género, que han sido publicitadas en mayor o menor medida en los últimos años, han tenido cierto seguimiento en las redacciones. Algunos decálogos, como el del periódico Público, han dado un paso más allá y se han comprometido ante su audiencia a cumplirlo.

Tercera: Asegurar el derecho de la ciudadanía a una información plural y veraz respecto a la igualdad de género, pasaría por:

Uno: La formación y competencia de quienes elaboran contenidos mediáticos y publicitarios.

Dos: Introducir en los planes de estudio de las facultades de ciencias de la información y la comunicación la perspectiva de género e incentivar dicha formación desde las estructuras de los propios medios.

Tres: Transformar la audiencia pasiva en un público crítico y exigente ante los medios de comunicación.

Cuatro: Es urgente que las mujeres, como grupo social significativo, hagan uso del derecho de acceso a la radio y la televisión de titularidad pública (tanto estatal como autonómica).

Cinco: La visibilización del estatus de quienes en el espacio público se comprometen con la igualdad es una buena medida para acercarse a ella.

Fecha de recepción: 30-04-2008

Fecha de aceptación: 07-05-2008 